\title{
The Transmuted Geometric-Inverse Weibull Distribution: Properties, Characterizations and Application
}

\author{
Fiaz Ahmad Bhatti \\ National College of Business Administration and Economics, Lahore, Pakistan. \\ Corresponding Author: fiazahmad72@gmail.com \\ D https://orcid.org/0000-0002-56 \\ Received: $31^{\text {st }}$ December 2017 / Revised: $03^{\text {rd }}$ of December 2018 / Published $07^{\text {th }}$ of December 2018: \\ CIAppStat-SL201
}

\begin{abstract}
In this paper, a four parameters flexible life time distribution called the transmuted geometric-inverse Weibull (TG-IW) distribution is obtained from mixture of inverse Weibull distribution, geometric distribution and transmuted distribution. Some structural and mathematical properties including descriptive measures on the basis of quantiles,moments, factorial moments, incomplete moments, inequality measures, residual life functions and some other properties are theoretically taken up.TheTGIW distribution is characterized via different techniques.The estimates of parametersfortheTG-IW distribution are being obtained from maximum likelihood method. The significance and flexibility of the TG-IW distribution is tested through different measures by application to physical data set.
\end{abstract}

Keywords: Geometric Distribution; Moments; Characterization; Maximum Likelihood Method;

\section{Introduction}

The generalizations of probability distribution are more flexible and suitable for many real data sets compared to classical distributions. Azzalini (1985) derived Skewed Family with additional skewing parameter. Gupta et al. (1998) developed exponentiated family. Marshal and Olkin (1997) introduced a parameter to a family of distributions. Eugene et al. (2002) established family formed from Beta distribution. Jones (2004) also presented a family generated from Beta distribution. The transmuted family was presented by Shaw and Buckley (2007).

Zografos Balakrishnan (2009) established family made from gamma distribution. Cordeiro and Castro (2011) developed family produced from Kumaraswamy 
distribution. Alexander et al. (2012) studied family made from McDonald distribution. Cordeiro et al. (2013) studied exponentiated generalized family of distribution. Torabi and Montazari (2014) studied a family of distributions created from logistic distribution. Alizadeh et. Al (2015) also developed Kumaraswamy Marshal Olkin family.Alizadeh et al. (2015) also proposed Kumaraswamy odd loglogistic family. Afify et.al (2016) developed a family of distributions named Kumaraswamy transmuted-G family. Afify et.al (2016) presented transmuted geometric-G family (TG-G). Nofal et.al (2017) studied mathematical properties, characterizations and regression models for the transmuted geometric Weibull distribution. Khan and Jan (2016) studied inverse Weibull geometric (IW-G) distribution. Bhatti (2017) studied characterizations of inverse Weibull geometric (I-W-G) distribution. Bhatti et al. (2018) studied the transmuted geometricquadratic hazard rate distribution along with its various properties.

The basic motivations for the TG-IW distribution is (i) to generate distributions with arc and positively skewed shaped (ii) to get increasing or decreasing and inverted bathtub hazard rate function (iii) to serve as the best alternative model for other current models to explore and modeling real data in economics, life testing, reliability, survival analysis manufacturing and other areas of research and (iv) to provides better fits than other sub-models.

Our interest is to study the TG-IW distribution along with its properties, applications and examine the usefulness of this distribution for modeling phenomena compared to the sub-models.

\section{The TG-IW Distribution}

The probability density function (pdf) for mixture of continuous probability distribution, geometric distribution and transmuted distribution is

$$
f(x ; \theta, \lambda)=\frac{\theta g(x)}{[1+(\theta-1) G(x)]^{2}}\left[1+\lambda-\frac{2 \lambda \theta G(x)}{(1+(\theta-1) G(x))}\right],|\lambda| \leq 1, \theta \in(0,1), x>0 .
$$

The cumulative distribution function (cdf) for TG-G family mixture of continuous probability distribution, geometric distribution and transmuted distribution is 


$$
F(x ; \theta, \lambda)=\frac{\theta G(x)}{[1+(\theta-1) G(x)]}\left\lfloor 1+\frac{\lambda \bar{G}(x)}{(1+(\theta-1) G(x))}\right\rfloor, \quad|\lambda| \leq 1, \theta \in(0,1), x \geq 0 .
$$

The popular lifetime distribution applicable for useful life, failure time spans, mortality, maintenance spans and maintenance cost in the fields like survival and reliability analysis are called inverse Weibull distributions. The cdf and pdf of inverse Weibull distributions are

$$
\mathrm{G}(x ; \alpha, \beta)=e^{-(\beta / x)^{\alpha}}, \quad x \geq 0,
$$

and

$$
\mathrm{g}(x ; k, \alpha, \beta)=\alpha \beta^{\alpha} x^{-\alpha-1} e^{-(\beta / x)^{\alpha}}, x>0 .
$$

The object of this article is to propose four parameters the TG-IW distribution from mixture of inverse Weibull distribution, geometric distribution and transmuted distribution by the application of Transmuted geometric-G family (TG-G).

The pdf and cdf of a random variable $\mathrm{X}$ with the TG-IW distribution are obtained by inducting (3a) and (3b) in (1) and (2) as

$$
\begin{aligned}
& f(x ; \alpha, \beta, \lambda, \theta)=\frac{\theta \alpha \beta^{\alpha} x^{-\alpha-1} e^{-(\beta / x)^{\alpha}}}{\left[1+(\theta-1) e^{-(\beta / x)^{\alpha}}\right]^{2}}\left\lfloor 1+\lambda-\frac{2 \lambda \theta e^{-(\beta / x)^{\alpha}}}{\left(1+(\theta-1) e^{-(\beta / x)^{\alpha}}\right)}\right], \quad \mathrm{x}>0, \\
& \left.F(x ; \alpha, \beta, \lambda, \theta)=\frac{\theta e^{-(\beta / x)^{\alpha}}}{\left[1+(\theta-1) e^{-(\beta / x)^{\alpha}}\right]} \mid 1+\frac{\lambda\left(1-e^{-(\beta / x)^{\alpha}}\right)}{\left(1+(\theta-1) e^{-(\beta / x)^{\alpha}}\right)}\right\rfloor, x \geq 0,
\end{aligned}
$$

where $\alpha>0, \beta>0,|\lambda| \leq 1$ and $\theta \in(0,1)$ are parameters. It is simple to observe that $F(x)$ is strictly increasing and differential in $(0, \infty)$. The cdf of the TG-IW also show that $\lim _{x \rightarrow \infty} F(x)=1$ and $\lim _{x \rightarrow 0} F(x)=0$. It means that $F(x)$ is an absolutely continuous cdf.

This research article is composed as follows. In Section 2, the TG-IW distribution is introduced. In Section 3, the TG-IW distribution is studied in terms of various 
structural properties, plots,sub-models and descriptive measures on the basis of quantiles are taken up. In Section 4, moments about origin, negative moments, fractional moments, moments about mean, moment generating function, cumulants generating function, incomplete moments, residual life functions, mean inactivity life and mean residual life function and inequality measures are and some other properties are theoretically derived. In Section 5, order statistics for the TG-IW distribution are proposed. In Section 6, the TG-IW distribution is characterized through (i) ratio of truncated moments; (ii) reverse hazard rate function and (iii) elasticity function. In Section 7, estimates of parameters for the TG-IW distribution are obtained from maximum likelihood method. Goodness of fit of the TG-IW distribution is checked through different methods is studied. Conclusion is given at the end.

\section{Structural Properties of the TG-IW Distribution}

The survival, hazard, reverse hazard and cumulative hazard functions of a random variable $\mathrm{X}$ with the TG-IW distribution are given, respectively, by

$$
\begin{aligned}
& f(x ; \alpha, \beta, \lambda, \theta)=\frac{\theta \alpha \beta^{\alpha} x^{-\alpha-1} e^{-(\beta / x)^{\alpha}}}{\left[1+(\theta-1) e^{-(\beta / x)^{\alpha}}\right]^{2}}\left\lfloor 1+\lambda-\frac{2 \lambda \theta e^{-(\beta / x)^{\alpha}}}{\left(1+(\theta-1) e^{-(\beta / x)^{\alpha}}\right)}\right], \quad \mathrm{x}>0, \\
& F(x ; \alpha, \beta, \lambda, \theta)=\frac{\theta e^{-(\beta / x)^{\alpha}}}{\left[1+(\theta-1) e^{-(\beta / x)^{\alpha}}\right]}\left[1+\frac{\lambda\left(1-e^{-(\beta / x)^{\alpha}}\right)}{\left(1+(\theta-1) e^{-(\beta / x)^{\alpha}}\right)}\right], x \geq 0, \\
& S(x)=1-\frac{\theta e^{-(\beta / x)^{\alpha}}}{\left(1+(\theta-1) e^{-(\beta / x)^{\alpha}}\right)}\left(1+\frac{\lambda\left(1-e^{-(\beta / x)^{\alpha}}\right)}{\left(1+(\theta-1) e^{\left.-(\beta / x)^{\alpha}\right)}\right)},(6)\right. \\
& h(x)=\frac{\theta \alpha \beta^{\alpha} x^{-\alpha-1} e^{-(\beta / x)^{\alpha}}\left\lfloor(1+\lambda)\left(1+(\theta-1) e^{-(\beta / x)^{\alpha}}\right)-2 \lambda \theta e^{-(\beta / x)^{\alpha}}\right]}{\left[1+(\theta-1) e^{-(\beta / x)^{\alpha}}\right]^{3}-\theta e^{-(\beta / x)^{\alpha}}\left[1+(\theta-1) e^{-(\beta / x)^{\alpha}}+\lambda\left(1-e^{-(\beta / x)^{\alpha}}\right)\right]},
\end{aligned}
$$


Sri Lankan Journal of Applied Statistics, Vol (18-3)

$H(x)=-\ln \left[1-\frac{\theta e^{-(\beta / x)^{\alpha}}}{\left(1+(\theta-1) e^{-(\beta / x)^{\alpha}}\right)}\left(1+\frac{\lambda\left(1-e^{-(\beta / x)^{\alpha}}\right)}{\left(1+(\theta-1) e^{-(\beta / x)^{\alpha}}\right)}\right)\right]$,

and

$r(x)=\frac{f(x)}{F(x)}=\frac{\alpha \beta^{\alpha} x^{-\alpha-1}\left(1+\lambda-\frac{2 \lambda \theta e^{-(\beta / x)^{\alpha}}}{\left(1+(\theta-1) e^{-(\beta / x)^{\alpha}}\right)}\right)}{\left[\left(1+(\theta-1) e^{-(\beta / x)^{\alpha}}\right)+\lambda\left(1-e^{-(\beta / x)^{\alpha}}\right)\right]}$,

The Mills ratio of the TG-IW distribution is

$$
m(x)=\frac{\left[1+(\theta-1) e^{-(\beta / x)^{\alpha}}\right]^{3}-\theta e^{-(\beta / x)^{\alpha}}\left[1+(\theta-1) e^{-(\beta / x)^{\alpha}}+\lambda\left(1-e^{-(\beta / x)^{\alpha}}\right)\right]}{\theta \alpha \beta^{\alpha} x^{-\alpha-1} e^{-(\beta / x)^{\alpha}}\left[(1+\lambda)\left(1+(\theta-1) e^{-(\beta / x)^{\alpha}}\right)-2 \lambda \theta e^{-(\beta / x)^{\alpha}}\right]} .
$$

The Elasticity $e(x)=\frac{d \ln \mathrm{F}(x)}{d \ln x}=x r(x)$, of the TG-IW distribution is

$$
e(x)=\frac{\alpha \beta^{\alpha}\left[(1+\lambda)\left(1+(\theta-1) e^{-(\beta / x)^{\alpha}}\right)-2 \lambda \theta e^{-(\beta / x)^{\alpha}}\right\rfloor}{x^{\alpha}\left[1+(\theta-1) e^{-(\beta / x)^{\alpha}}+\lambda\left(1-e^{-(\beta / x)^{\alpha}}\right)\right]} .
$$

The elasticity of the TG-IW distribution shows the behavior of accumulation of probability in the domain of the random variable.

\subsection{Shapes of the TG-IW Density and Hazard Rate Function}

The TG-IW density is arc and positively skewed (Fig. 1). The TG-IW hazard is increasing or decreasing and inverted bathtub hazard rate function (Fig. 2). 
Sri Lankan Journal of Applied Statistics, Vol (18-3)

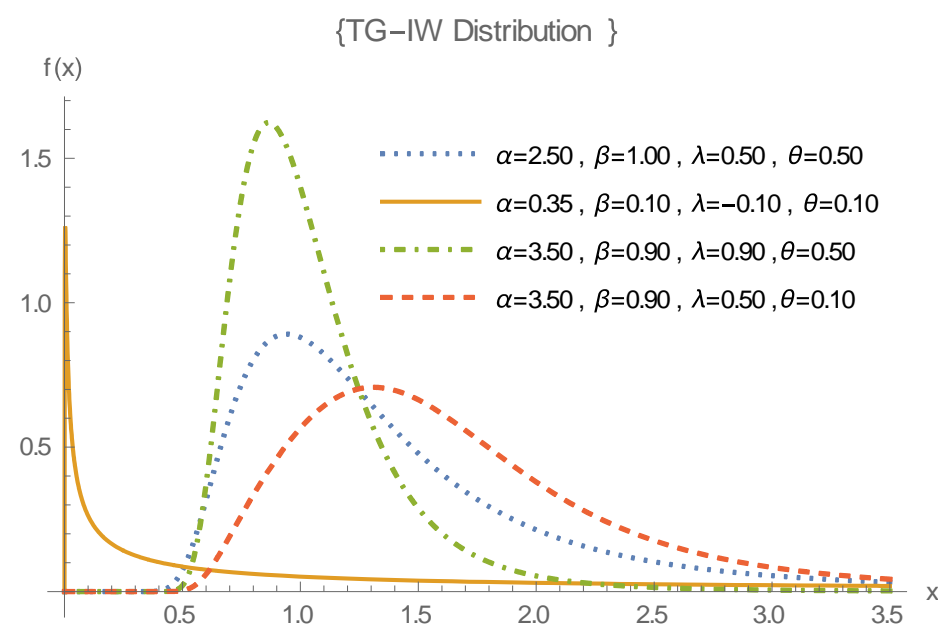

Fig. 1: Plots of pdf of the TG-IW Distribution

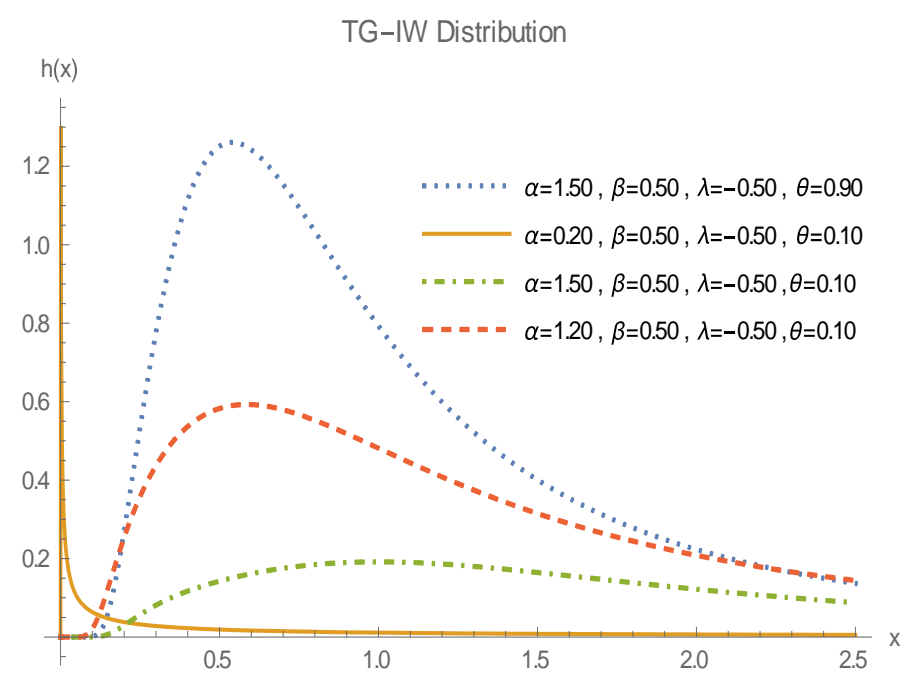

Fig. 2: Plots of hrf of the TG-IW Distribution

\subsection{Sub Models of the TG-IW Distribution}

The TG-IW distribution has wide applications in life testing, survival analysis, and reliability theory. The TG-IW distribution has the following sub models. 
Sri Lankan Journal of Applied Statistics, Vol (18-3)

Table 1: Sub-models of the TG-IW Distribution

\begin{tabular}{|l|l|l|l|l|l|}
\hline Sr.No. & $\theta$ & $\lambda$ & $\alpha$ & $\beta$ & Name of Distribution \\
\hline 1 & 0 & 0 & 1 & $\beta$ & Inverse Exponential \\
\hline 2 & 0 & 0 & 2 & $\beta$ & Inverse Rayleigh \\
\hline 3 & 0 & 0 & $\alpha$ & $\beta$ & Inverse Weibull \\
\hline 4 & $\theta$ & 0 & 1 & $\beta$ & $\begin{array}{l}\text { Inverse Exponential } \\
\text { Geometric }\end{array}$ \\
\hline 5 & $\theta$ & 0 & 2 & $\beta$ & Inverse Rayleigh Geometric \\
\hline 6 & $\theta$ & 0 & $\alpha$ & $\beta$ & Inverse Weibull Geometric \\
\hline 7 & 0 & $\lambda$ & 1 & $\beta$ & $\begin{array}{l}\text { Transmuted Inverse } \\
\text { Exponential }\end{array}$ \\
\hline 8 & 0 & $\lambda$ & 2 & $\beta$ & Transmuted Inverse Rayleigh \\
\hline 9 & 0 & $\lambda$ & $\alpha$ & $\beta$ & Transmuted Inverse Weibull \\
\hline 10 & $\theta$ & $\lambda$ & 1 & $\beta$ & TG-Inverse Exponential \\
\hline 11 & $\theta$ & $\lambda$ & 2 & $\beta$ & TG-Inverse Rayleigh \\
\hline 12 & $\theta$ & $\lambda$ & $\alpha$ & $\beta$ & TG-Inverse Weibull \\
\hline
\end{tabular}

\subsection{Descriptive Measures Based On Quantiles}

In this sub-section, descriptive measures on the basis of quantiles are taken up.

The quantile function of the TG-IW distribution is

$$
x_{q}=\left\{-\frac{1}{\beta^{\alpha}} \ln \left[\frac{\lambda+1-\sqrt{(\lambda+1)^{2}-4 \lambda q}}{2 \lambda \theta+(1-\theta)\left[\lambda+1-\sqrt{(\lambda+1)^{2}-4 \lambda q}\right]}\right]\right\}^{-\frac{1}{\alpha}} .
$$

Median of the TG-IW distribution is

$$
\left.x_{\text {Med }}=\left\{-\frac{1}{\beta^{\alpha}} \ln \left[\frac{\lambda+1-\sqrt{(\lambda+1)^{2}-2 \lambda}}{2 \lambda \theta+(1-\theta)\left[\lambda+1-\sqrt{(\lambda+1)^{2}-2 \lambda}\right.}\right]\right]\right\}^{-\frac{1}{\alpha}} .
$$


Sri Lankan Journal of Applied Statistics, Vol (18-3)

the random number generator of the TG-IW distribution is

$$
X=\left\{-\frac{1}{\beta^{\alpha}} \ln \left[\frac{\lambda+1-\sqrt{(\lambda+1)^{2}-4 \lambda Z}}{2 \lambda \theta+(1-\theta)\left[\lambda+1-\sqrt{(\lambda+1)^{2}-4 \lambda Z}\right]}\right]\right\}^{-\frac{1}{\alpha}} .
$$

where the random variable $\mathrm{Z}$ has uniform distribution on $(0,1)$. Some measures based

on quartiles for location, dispersion, skewness and kurtosis for the TG-IW distribution respectively are: Median $\mathrm{M}=\mathrm{Q}$ (0.5) and quartile deviation is

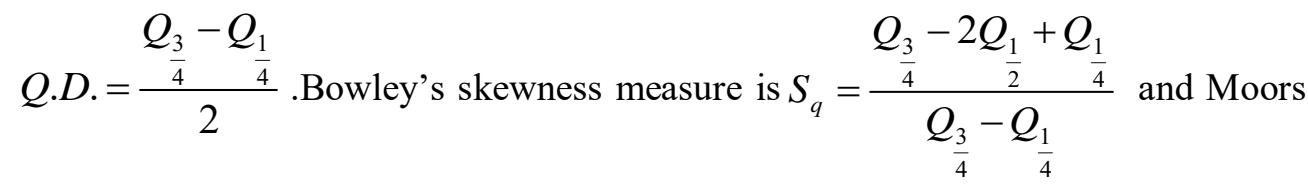
kurtosis measure based on Octiles is $K=\frac{\frac{Q_{7}}{8}-Q_{\frac{5}{8}}+Q_{\frac{3}{8}}-Q_{\frac{1}{8}}}{Q_{\frac{6}{8}}-Q_{\frac{2}{8}}}$. The measures based on quantile exist for the distributions whose moments does not exist. The measures based on quantiles are rarer sensitive to the outliers.

\subsection{Median Inactivity Time Function}

Kandil et al.(2010) developed median inactivity time (MDIT) function. The MDIT function in terms of cdf of a continuous life time distribution is $\operatorname{MDIT}(z)=z-F_{X}^{-1}\left(\frac{1}{2} F_{X}(z)\right)$.

The MDIT function in terms of (5) for the TG-IW distribution is

$$
\operatorname{MDIT}(z)=z-\left\{-\frac{1}{\beta^{\alpha}} \ln \left[\frac{\lambda+1-\sqrt{(\lambda+1)^{2}-\frac{2 \lambda \theta e^{-(\beta / z)^{\alpha}}}{\left[1+(\theta-1) e^{-(\beta / z)^{\alpha}}\right]\left[1+\frac{\lambda\left(1-e^{-(\beta / z)^{\alpha}}\right)}{\left(1+(\theta-1) e^{-(\beta / z)^{\alpha}}\right)}\right]}}}{2 \lambda \theta+(1-\theta)\left[\lambda+1-\sqrt{\left.(\lambda+1)^{2}-\frac{2 \lambda \theta e^{-(\beta / z)^{\alpha}}}{\left[1+(\theta-1) e^{-(\beta / z)^{\alpha}}\right]\left[1+\frac{\lambda\left(1-e^{-(\beta / z)^{\alpha}}\right)}{\left(1+(\theta-1) e^{-(\beta / z)^{\alpha}}\right)}\right]}\right]}\right]} .\right.\right.
$$


Sri Lankan Journal of Applied Statistics, Vol (18-3)

\section{Moments and Inequality Measures}

In this section, moments about origin, negative moments, fractional moments, moment about mean, moment generating function, cumulants generating function, incomplete moments, inequality measures, residual life functions, mean inactivity life, mean residual life function and some other properties are theoretically derived.

\subsection{Moments about origin}

The $\mathrm{r}^{\text {th }}$ moments of the random variable $\mathrm{X}$ with the TG-IW distribution about the origin are given by

$$
\begin{aligned}
& \mu_{r}^{\prime}=E\left(X^{r}\right)=\int_{0}^{\infty} x^{r} \frac{\theta \alpha \beta^{\alpha} x^{-\alpha-1} e^{-(\beta / x)^{\alpha}}}{\left[1+(\theta-1) e^{-(\beta / x)^{\alpha}}\right]^{2}}\left[1+\lambda-\frac{2 \lambda \theta e^{-(\beta / x)^{\alpha}}}{\left(1+(\theta-1) e^{-(\beta / x)^{\alpha}}\right)}\right] d x, \\
& \mu_{r}^{\prime}=\beta^{r} \theta \Gamma\left(1-\frac{r}{\alpha}\right) \sum_{\ell=0}^{\infty} \frac{(-1)^{\ell}}{\ell !}(\theta-1)^{\ell}\left[(1+\lambda)(\ell+1)^{\frac{r}{\alpha}-1}(2)_{\ell}-2 \lambda \theta(\ell+2)^{\frac{r}{\alpha}-1}(3)_{\ell}\right],
\end{aligned}
$$

where $(\ell)_{k}=\frac{\Gamma(\ell+k)}{\Gamma(\ell)}$ is Pochhammar symbol.

Mean and Variance for the TG-IW distribution are given, respectively

$$
E(X)=\beta \theta \Gamma\left(1-\frac{1}{\alpha}\right) \sum_{\ell=0}^{\infty} \frac{(-1)^{\ell}}{\ell !}(\theta-1)^{\ell}\left[(1+\lambda)(\ell+1)^{\frac{1}{\alpha}-1}(2)_{\ell}-2 \lambda \theta(\ell+2)^{\frac{1}{\alpha}-1}(3)_{\ell}\right] \text {, }
$$

and

$$
\operatorname{Var}(X)=\left[\beta^{2} \theta \Gamma\left(1-\frac{2}{\alpha}\right) \sum_{\ell=0}^{\infty} \frac{(-1)^{\ell}}{\ell !}(\theta-1)^{\ell}\left(\begin{array}{l}
(1+\lambda)(\ell+1)^{\frac{2}{\alpha}-1}(2)_{\ell}- \\
2 \lambda \theta(\ell+2)^{\frac{2}{\alpha}-1}(3)_{\ell}
\end{array}\right)-\left(\beta \Gamma\left(1-\frac{1}{\alpha}\right) \sum_{\ell=0}^{\infty} \frac{(-1)^{\ell}}{\ell !}(\theta-1)^{\ell}\left(\begin{array}{l}
\left.\left.(1+\lambda)(\ell+1)^{\frac{1}{\alpha}-1}(2)_{\ell}-\right)\right)^{2} \\
2 \lambda \theta(\ell+2)^{\frac{1}{\alpha}-1}(3)_{\ell}
\end{array}\right)\right) .\right.
$$

An important measure of variability of a random variable is Fisher index of dispersion 
Sri Lankan Journal of Applied Statistics, Vol (18-3)

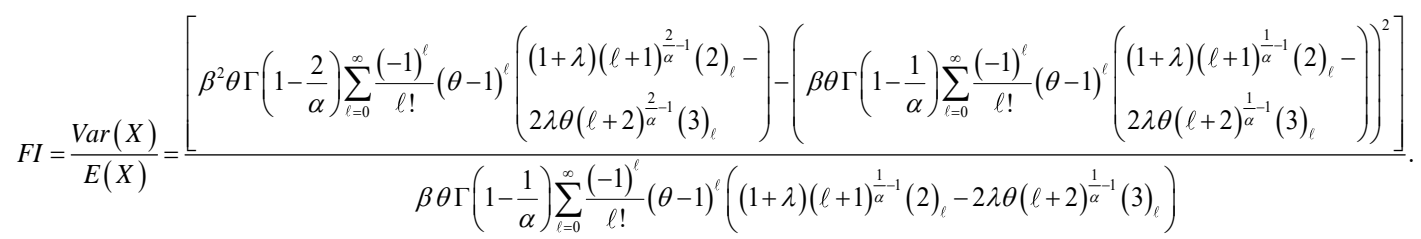

For $\mathrm{FI}=1$, the TG-IW distribution is equidispersed, for $\mathrm{FI}<1$, the TG-IW distribution is under dispersed and for FI $>1$, the TG-IW distribution is over dispersed.

The fractional positive moments of $\mathrm{X}$ with the TG-IW distribution about the origin are given by

$$
\mu_{\frac{r}{s}}^{\prime}=E\left(X^{\frac{r}{s}}\right)=\beta^{\frac{r}{s}} \theta \Gamma\left(1-\frac{r}{\alpha s}\right) \sum_{\ell=0}^{\infty} \frac{(-1)^{\ell}}{\ell !}(\theta-1)^{\ell}\left[(1+\lambda)(\ell+1)^{\frac{r}{\alpha s}-1}(2)_{\ell}-2 \lambda \theta(\ell+2)^{\frac{r}{\alpha s}-1}(3)_{\ell}\right] .
$$

The $\mathrm{q}^{\text {th }}$ central moments about mean of $\mathrm{X}$ for the TG-IW distribution are determined from

$$
\begin{gathered}
\mu_{q}=E[X-E(X)]^{q}=\sum_{v=1}^{q}\left(\begin{array}{c}
q \\
v
\end{array}\right)(-1)^{v} E\left(X^{v}\right) E\left(X^{q-v}\right), \\
\mu_{q}=\beta^{q} \theta^{2} \sum_{v=1}^{q}\left(\begin{array}{l}
q \\
v
\end{array}\right)(-1)^{v} \Gamma\left(1-\frac{v}{\alpha}\right) \Gamma\left(1-\frac{q-v}{\alpha}\right)\left(\begin{array}{l}
(1+\lambda) \sum_{\ell=0}^{\infty} \frac{(-1)^{\ell}}{\ell !}(\theta-1)^{\ell}(\ell+1)^{\frac{v}{\alpha}-1}(2)_{\ell}- \\
2 \lambda \theta \sum_{\ell=0}^{\infty} \frac{(-1)^{\ell}}{\ell !}(\theta-1)^{\ell}(\ell+2)^{\frac{v}{\alpha}-1}(3)_{\ell}
\end{array}\right)\left(\begin{array}{l}
(1+\lambda) \sum_{\ell=0}^{\infty} \frac{(-1)^{\ell}}{\ell !}(\theta-1)^{\ell}(\ell+1)^{\frac{(q-v)}{\alpha}-1}(2)_{\ell}- \\
2 \lambda \theta \sum_{\ell=0}^{\infty} \frac{(-1)^{\ell}}{\ell !}(\theta-1)^{\ell}(\ell+2)^{\frac{(q-v)}{\alpha}-1}(3)_{\ell}
\end{array}\right) .
\end{gathered}
$$


Sri Lankan Journal of Applied Statistics, Vol (18-3)

Pearson's measures of skewness $\gamma_{1}$ and Kurtosis $\beta_{2}$ are

$$
\gamma_{1}=\frac{\mu_{3}}{\left(\mu_{2}\right)^{\frac{3}{2}}}=\frac{\left(\beta^{3} \theta^{2} \sum_{v=1}^{3}\left(\begin{array}{l}
3 \\
v
\end{array}\right)(-1)^{v} \Gamma\left(1-\frac{v}{\alpha}\right) \Gamma\left(1-\frac{3-v}{\alpha}\right)\left(\begin{array}{c}
(1+\lambda) \sum_{\ell=0}^{\infty} \frac{(-1)^{\ell}}{\ell !}(\theta-1)^{\ell}(\ell+1)^{\frac{v}{\alpha}-1}(2)_{\ell}- \\
2 \lambda \theta \sum_{\ell=0}^{\infty} \frac{(-1)^{\ell}}{\ell !}(\theta-1)^{k}(\ell+2)^{\frac{v}{\alpha}-1}(3)_{\ell}
\end{array}\right)\left(\begin{array}{c}
\left.(1+\lambda) \sum_{\ell=0}^{\infty} \frac{(-1)^{\ell}}{\ell !}(\theta-1)^{\ell}(\ell+1)^{\frac{3-v}{\alpha}-1}(2)_{\ell}-\right) \\
2 \lambda \theta \sum_{\ell=0}^{\infty} \frac{(-1)^{\ell}}{\ell !}(\theta-1)^{k}(\ell+2)^{\frac{3-v}{\alpha}-1}(3)_{\ell}
\end{array}\right)\right]}{\left.\beta^{2} \theta^{2} \sum_{v=1}^{2}\left(\begin{array}{l}
2 \\
v
\end{array}\right)(-1)^{v} \Gamma\left(1-\frac{v}{\alpha}\right) \Gamma\left(1-\frac{2-v}{\alpha}\right)\left(\begin{array}{c}
(1+\lambda) \sum_{\ell=0}^{\infty} \frac{(-1)^{\ell}}{\ell !}(\theta-1)^{\ell}(\ell+1)^{\frac{v}{\alpha}-1}(2)_{\ell} \\
2 \lambda \theta \sum_{\ell=0}^{\infty} \frac{(-1)^{\ell}}{\ell !}(\theta-1)^{k}(\ell+2)^{\frac{v}{\alpha}-1}(3)_{\ell}
\end{array}\right)\left(\begin{array}{l}
(1+\lambda) \sum_{\ell=0}^{\infty} \frac{(-1)^{\ell}}{\ell !}(\theta-1)^{\ell}(\ell+1)^{\frac{2-v}{\alpha}-1}(2)_{\ell}- \\
2 \lambda \theta \sum_{\ell=0}^{\infty} \frac{(-1)^{\ell}}{\ell !}(\theta-1)^{\ell}(\ell+2)^{\frac{2-v}{\alpha}-1}(3)_{\ell}
\end{array}\right)\right]}
$$

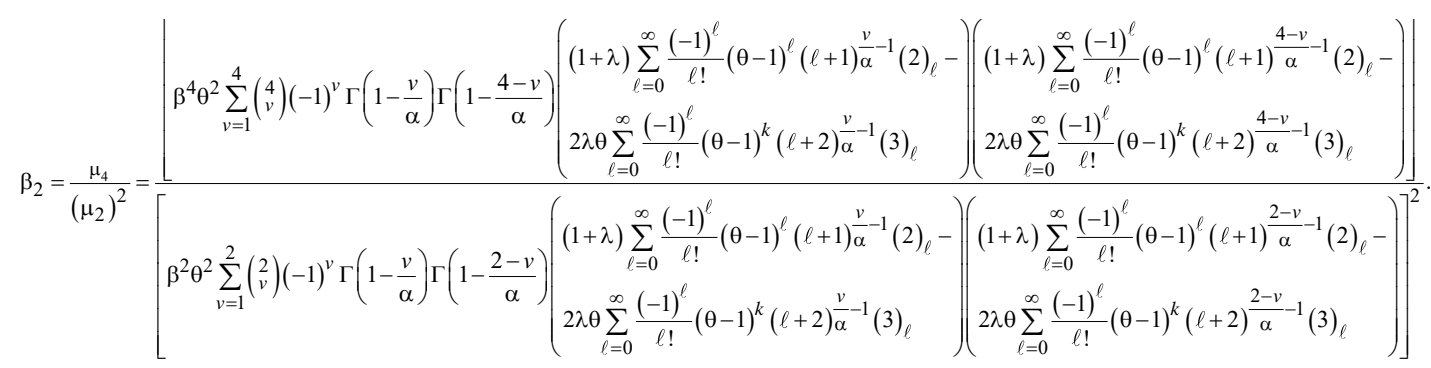

The negative moments are used to determine to harmonic mean and many other measures. The $r^{\text {th }}$ negative moments about origin of $X$ for the TG-IW distribution are

$$
\mu_{-r}^{\prime}=E\left(X^{-r}\right)=\beta^{-r} \theta \Gamma\left(1+\frac{r}{\alpha}\right) \sum_{\ell=0}^{\infty} \frac{(-1)^{\ell}}{\ell !}(\theta-1)^{\ell}\left[(1+\lambda)(\ell+1)^{-\frac{r}{\alpha}-1}(2)_{\ell}-2 \lambda \theta(\ell+2)^{-\frac{r}{\alpha}-1}(3)_{\ell}\right] .
$$

The fractional negative moments about origin of $\mathrm{X}$ for the TG-IW distribution are given as

$\mu_{-\frac{r}{s}}^{\prime}=E\left(X^{-\frac{r}{s}}\right)=\beta^{\frac{r}{s}} \theta \Gamma\left(1-\frac{r}{\alpha s}\right) \sum_{\ell=0}^{\infty} \frac{(-1)^{\ell}}{\ell !}(\theta-1)^{\ell}\left[(1+\lambda)(\ell+1)^{\frac{r}{\alpha s}-1}(2)_{\ell}-2 \lambda \theta(\ell+2)^{\frac{r}{\alpha s}-1}(3)_{\ell}\right]$. 
Sri Lankan Journal of Applied Statistics, Vol (18-3)

The factorial moments for the TG-IW distribution are

$$
\begin{gathered}
E[X]_{m}=\sum_{r=1}^{m} \gamma_{r} E\left(X^{r}\right)=\sum_{r=1}^{m} \gamma_{r} \mu_{r}^{\prime}, \\
E[X]_{m}=\sum_{r=1}^{n} \gamma_{r} \beta^{r} \theta \Gamma\left(1-\frac{r}{\alpha}\right) \sum_{\ell=0}^{\infty} \frac{(-1)^{\ell}}{\ell !}(\theta-1)^{\ell}\left[(1+\lambda)(\ell+1)^{\frac{r}{\alpha}-1}(2)_{\ell}-2 \lambda \theta(\ell+2)^{\frac{r}{\alpha}-1}(3)_{\ell}\right],
\end{gathered}
$$

where $[Z]_{i}=Z(Z+1)(Z+2) \ldots \ldots(Z+i-1)$ and $\gamma_{r}$ is Stirling number of the first kind.

The Mellin transform helps to determine moments for a probability distribution. By definition, the Mellin transform is

$$
\mathrm{M}\{f(x) ; s\}=f^{*}(s)=\int_{0}^{\infty} f(x) x^{s-1} d x .
$$

The Mellin transform of $\mathrm{X}$ for the TG-IW distribution is

$$
\begin{aligned}
& M\{f(x) ; s\}=\int_{0}^{\infty} x^{s-1} \frac{\theta \alpha \beta^{\alpha} x^{-\alpha-1} e^{-(\beta / x)^{\alpha}}}{\left[1+(\theta-1) e^{-(\beta / x)^{\alpha}}\right]^{2}}\left[\begin{array}{l}
2 \lambda \theta e^{-(\beta / x)^{\alpha}} \\
\left(1+(\theta-1) e^{-(\beta / x)^{\alpha}}\right)
\end{array} d x,\right. \\
& M\{f(x) ; s\}=\beta^{s-1} \theta \Gamma\left(1-\frac{s-1}{\alpha}\right) \sum_{\ell=0}^{\infty} \frac{(-1)^{\ell}}{\ell !}(\theta-1)^{\ell}\left[(1+\lambda)(\ell+1) \frac{s-1}{\alpha}-1(2)_{\ell}-2 \lambda \theta(\ell+2)^{\frac{s-1}{\alpha}-1}(3)_{\ell}\right] .
\end{aligned}
$$

The moments generating function of random variable $\mathrm{X}$ with the TG-IW distribution is

$$
\begin{gathered}
M_{X}(t)=E\left[e^{t X}\right]=\sum_{r=0}^{r} \frac{t^{r}}{r !} \mu_{r}^{\prime}, \\
M_{x}(t)=\sum_{r=0}^{r} \frac{t^{r}}{r !} \beta^{r} \theta \Gamma\left(1-\frac{r}{\alpha}\right) \sum_{\ell=0}^{\infty} \frac{(-1)^{\ell}}{\ell !}(\theta-1)^{\ell}\left[(1+\lambda)(\ell+1)^{\frac{r}{\alpha}-1}(2)_{\ell}-2 \lambda \theta(\ell+2)^{\frac{r}{\alpha}-1}(3)_{\ell}\right] .
\end{gathered}
$$


Sri Lankan Journal of Applied Statistics, Vol (18-3)

The cumulant moments generating function $K(t)$ for $\mathrm{X}$ with the TG-IW distribution is

$$
\begin{gathered}
\mathrm{K}(t)=\log M_{X}(t)=\log \left\lfloor\sum_{r=0}^{r} \frac{t^{r}}{r !} \mu_{r}^{\prime}\right\rfloor \\
\mathrm{K}(t)=\log \left\lfloor\sum_{r=0}^{r} \frac{t^{r}}{r !} \beta^{r} \theta \Gamma\left(1-\frac{r}{\alpha}\right) \sum_{\ell=0}^{\infty} \frac{(-1)^{\ell}}{\ell !}(\theta-1)^{\ell}\left((1+\lambda)(\ell+1)^{\frac{r}{\alpha}-1}(2)_{\ell}-2 \lambda \theta(\ell+2)^{\frac{r}{\alpha}-1}(3)_{\ell}\right)\right\rfloor .
\end{gathered}
$$

The cumulants for $\mathrm{X}$ with the TG-IW distribution are obtained from relation

$$
\begin{aligned}
& k_{r}=E\left(X^{r}\right)-\sum_{c=1}^{r-1}\left(\begin{array}{c}
r-1 \\
c-1
\end{array}\right) k_{c} E\left(X^{r-c}\right), \text { as } \\
& k_{r}=\left\{\begin{array}{l}
\beta^{r} \theta \Gamma\left(1-\frac{r}{\alpha}\right) \sum_{\ell=0}^{\infty} \frac{(-1)^{\ell}}{\ell !}(\theta-1)^{\ell}\left((1+\lambda)(\ell+1)^{\frac{r}{\alpha}-1}(2)_{\ell}-2 \lambda \theta(\ell+2)^{\frac{r}{\alpha}-1}(3)_{\ell}\right) \\
\left.-\sum_{c=1}^{r-1}\left(\begin{array}{c}
r-1 \\
c-1
\end{array}\right) k_{c} \beta^{r-c} \theta \Gamma\left(1-\frac{r}{\alpha}\right) \sum_{\ell=0}^{\infty} \frac{(-1)^{\ell}}{\ell !}(\theta-1)^{\ell}\left((1+\lambda)(\ell+1)^{\frac{r-c}{\alpha}-1}(2)_{\ell}-2 \lambda \theta(\ell+2)^{\frac{r-c}{\alpha}-1}(3)_{\ell}\right)\right\} .
\end{array}\right.
\end{aligned}
$$

\subsection{Incomplete Moments}

Incomplete moments are used to study mean inactivity life, mean residual life function and other inequality measures.

The lower incomplete moments for the random variable $\mathrm{X}$ with the TG-IW distribution are

$$
\begin{gathered}
\varphi_{r}(z)=E_{X \leq z}\left(X^{r}\right)=\int_{0}^{z} x^{r} \frac{\theta \alpha \beta^{\alpha} x^{-\alpha-1} e^{-(\beta / x)^{\alpha}}}{\left[1+(\theta-1) e^{-(\beta / x)^{\alpha}}\right]^{2}}\left[1+\lambda-\frac{2 \lambda \theta e^{-(\beta / x)^{\alpha}}}{\left(1+(\theta-1) e^{-(\beta / x)^{\alpha}}\right)}\right] d x, \\
\varphi_{r}(z)=\beta^{r} \theta \gamma\left(z ; 1-\frac{r}{\alpha}\right) \sum_{\ell=0}^{\infty} \frac{(-1)^{\ell}}{\ell !}(\theta-1)^{\ell}\left[(1+\lambda)(\ell+1)^{\frac{r}{\alpha}-1}(2)_{\ell}-2 \lambda \theta(\ell+2)^{\frac{r}{\alpha}-1}(3)_{\ell}\right] .
\end{gathered}
$$


Sri Lankan Journal of Applied Statistics, Vol (18-3)

The upper incomplete moments for the random variable $\mathrm{X}$ with the TG-IW distribution are

$$
\begin{aligned}
& E_{X>z}\left(X^{r}\right)=\int_{z}^{\infty} x^{r} \frac{\theta \alpha \beta^{\alpha} x^{-\alpha-1} e^{-(\beta / x)^{\alpha}}}{\left[1+(\theta-1) e^{-(\beta / x)^{\alpha}}\right]^{2}}\left\lfloor 1+\lambda-\frac{2 \lambda \theta e^{-(\beta / x)^{\alpha}}}{\left(1+(\theta-1) e^{-(\beta / x)^{\alpha}}\right)}\right] d x, \\
& E_{X>z}\left(X^{r}\right)=\beta^{r} \theta \sum_{\ell=0}^{\infty} \frac{(-1)^{\ell}}{\ell !}(\theta-1)^{\ell}\left[(1+\lambda)(\ell+1)^{\frac{r}{\alpha}-1}(2)_{\ell}-2 \lambda \theta(\ell+2)^{\frac{r}{\alpha}-1}(3)_{\ell}\right]\left[\Gamma\left(1-\frac{r}{\alpha}\right)-\gamma\left(z ; 1-\frac{r}{\alpha}\right)\right] .
\end{aligned}
$$

The mean deviation about mean is $M D_{\bar{X}}=E\left|X-\mu_{1}^{1}\right|=2 \mu_{1}^{1} F\left(\mu_{1}^{1}\right)-2 \mu_{1}^{1} \varphi_{1}\left(\mu_{1}^{1}\right)$ and the mean deviation about median is $M D_{M}=E|X-M|=2 M F(M)-2 M \varphi_{1}(M)$ where $\mu_{1}^{\prime}=E(X)$, and $M=Q(0.5)$. Bonferroni and Lorenz curve for a specified probability $\mathrm{p}$ are $\mathrm{B}(p)=\varphi_{1}(q) / p \mu_{1}^{1}$ and $L(p)=\varphi_{1}(q) / \mu_{1}^{1}$ where $q=Q(p)$.

\subsection{Residual Life Functions}

The residual life says $m_{n}(z)$ of $\mathrm{X}$ for the TG-IW distribution having the following $\mathrm{n}^{\text {th }}$ moment

$$
\begin{gathered}
m_{n}(z)=E\left[(X-z)^{n} \mid X>z\right] \\
m_{n}(z)=\frac{1}{S(z)} \int_{z}^{\infty}(x-z)^{s} f(x) d x \\
m_{n}(z)=\frac{1}{S(z)} \sum_{s=0}^{n}\left(\begin{array}{l}
n \\
s
\end{array}\right)(-z)^{n-s} E_{X>z}\left(X^{s}\right) \\
m_{n}(z)=\frac{1}{S(z)} \sum_{s=0}^{n}\left(\begin{array}{l}
n \\
s
\end{array}\right)(-z)^{n-s} \beta^{s} \theta \sum_{\ell=0}^{\infty} \frac{(-1)^{\ell}}{\ell !}(\theta-1)^{\ell}\left[(1+\lambda)(\ell+1)^{\frac{s}{\alpha}-1}(2)_{\ell}-2 \lambda \theta(\ell+2)^{\frac{s}{\alpha}-1}(3)\right]\left[\Gamma\left(1-\frac{s}{\alpha}\right)-\gamma\left(z ; 1-\frac{s}{\alpha}\right)\right] \cdot
\end{gathered}
$$


Sri Lankan Journal of Applied Statistics, Vol (18-3)

The mean residual life function or life expectancy at a specified time $z$, say $m_{1}(z)$, computes the expected left over lifetime of an individual of age $\mathrm{z}$ and is

$$
m_{1}(z)=\frac{1}{S(z)} \sum_{s=0}^{1}\left(\begin{array}{l}
1 \\
s
\end{array}\right)(-z)^{1-s} \beta^{s} \theta \sum_{\ell=0}^{\infty} \frac{(-1)^{\ell}}{\ell !}(\theta-1)^{\ell}\left[(1+\lambda)(\ell+1)^{\frac{s}{\alpha}-1}(2)_{\ell}-2 \lambda \theta(\ell+2)^{\frac{s}{\alpha}-1}(3)_{\ell}\right]\left[\Gamma\left(1-\frac{s}{\alpha}\right)-\gamma\left(z ; 1-\frac{s}{\alpha}\right)\right] \text {. }
$$

The reverse residual life, say $M_{n}(z)$, of $\mathrm{X}$ for the TG-IW distribution has the following $\mathrm{n}^{\text {th }}$ moment

$$
\begin{gathered}
M_{n}(z)=E\left\lfloor(z-X)^{n} / X \leq z\right\rfloor \\
M_{n}(z)=\frac{1}{F(z)} \int_{a}^{z}(z-x)^{n} f(x) d x \\
M_{n}(z)=\frac{1}{F(z)} \sum_{s=0}^{n}(-1)^{s}\left(\begin{array}{l}
n \\
s
\end{array}\right) z^{n-s} E_{X \leq z}\left(X^{s}\right), \\
M_{n}(z)=\frac{1}{F(z)} \sum_{s=0}^{n}(-1)^{s}\left(\begin{array}{l}
n \\
s
\end{array}\right) z^{n-s} \beta^{s} \theta \gamma\left(z ; 1-\frac{s}{\alpha}\right) \sum_{\ell=0}^{\infty} \frac{(-1)^{\ell}}{\ell !}(\theta-1)^{\ell}\left[(1+\lambda)(\ell+1)^{\frac{s}{\alpha}-1}(2)_{\ell}-2 \lambda \theta(\ell+2)^{\frac{s}{\alpha}-1}(3)_{\ell}\right] .
\end{gathered}
$$

The mean waiting time (MWT) or mean inactivity time signifies the waiting time pass by since the failure of an item on condition that this failure had happened in the interval $[0, \mathrm{z}]$. The MWT of X, say $M_{1}(z)$, is defined by

$$
M_{1}(z)=\frac{1}{F(z)} \sum_{s=0}^{1}(-1)^{s}\left(\begin{array}{l}
1 \\
s
\end{array}\right) z^{1-s s} \theta \gamma\left(z ; 1-\frac{s}{\alpha}\right) \sum_{\ell=0}^{\infty} \frac{(-1)^{\ell}}{\ell !}(\theta-1)^{\ell}\left[(1+\lambda)(\ell+1)^{\frac{s}{\alpha}-1}(2)_{\ell}-2 \lambda \theta(\ell+2)^{\frac{s}{\alpha}-1}(3)_{\ell}\right]
$$

\section{Order Statistics}

The order statistics mostly appear in the problems of the estimation and testing. The application of extreme values is very common in reliability, meteorology, econometrics and various areas of research. 
Sri Lankan Journal of Applied Statistics, Vol (18-3)

The pdf $f_{X_{j: n}}(x)$ of $j$ th order statistic $X_{\mathrm{j}: n}$, from a cdf F with pdf $\mathrm{f}$, is

$$
f_{X_{j: n}}(x)=\frac{1}{B(\mathrm{j}, n-j+1)}[F(x)]^{j-1}[1-F(x)]^{n-j} f(x) .
$$

The pdf $f_{X_{j: n}}(x)$ of $j$ th order statistic $X_{\mathrm{j}: n}$ for the TG-IW distribution is given by

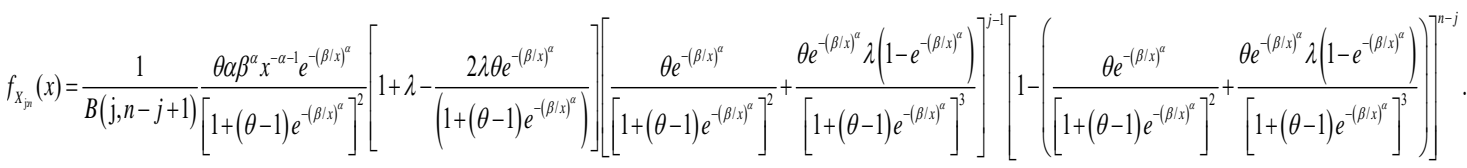

The pdf $f_{X_{n: n}}(x)$ of $n t h$ order statistic $X_{\mathrm{n}: n}$, from a cdf F with pdf $\mathrm{f}$, is

$$
f_{X_{n: n}}(x)=n[F(x)]^{n-1} f(x) .
$$

The pdf $f_{X_{n: n}}(x)$ of $n$th order statistic $X_{n: n}$ for the TG-IW distribution is given by

$$
f_{X_{\mathrm{n} n}}(x)=\frac{n \alpha \beta^{\alpha} \theta x^{-\alpha-1} e^{-(\beta / x)^{\alpha}}}{\left[1+(\theta-1) e^{-(\beta / x)^{\alpha}}\right]^{2}}\left[1+\lambda-\frac{2 \lambda \theta e^{-(\beta / x)^{\alpha}}}{\left(1+(\theta-1) e^{-(\beta / x)^{\alpha}}\right)}\right]\left[\frac{\theta e^{-(\beta / x)^{\alpha}}}{\left[1+(\theta-1) e^{-(\beta / x)^{\alpha}}\right]^{2}}+\frac{\theta e^{-(\beta / x)^{\alpha}} \lambda\left(1-e^{-(\beta / x)^{\alpha}}\right)}{\left[1+(\theta-1) e^{-(\beta / x)^{\alpha}}\right]^{3}}\right]^{n-1} .
$$

The pdf $f_{X_{1: n}}(x)$ of $1 s t$ order statistic $X_{1: n}$, from a cdf $\mathrm{F}$ with pdf $\mathrm{f}$, is

$$
f_{X_{1: n}}(x)=n[1-F(x)]^{n-1} f(x) .
$$

The pdf $f_{X_{1: n}}(x)$ of $1 s t$ order statistic $X_{1: n}$ for the TG-IW distribution is given by

$$
f_{X_{\mathrm{ln}}}(x)=\frac{n \alpha \beta^{\alpha} \theta x^{-\alpha-1} e^{-(\beta / x)^{\alpha}}}{\left[1+(\theta-1) e^{-(\beta / x)^{\alpha}}\right]^{2}}\left[1+\lambda-\frac{2 \lambda \theta e^{-(\beta / x)^{\alpha}}}{\left(1+(\theta-1) e^{-(\beta / x)^{\alpha}}\right)}\right]\left[1-\left(\frac{\theta e^{-(\beta / x)^{\alpha}}}{\left[1+(\theta-1) e^{-(\beta / x)^{\alpha}}\right]^{2}}+\frac{\theta e^{-(\beta / x)^{\alpha}} \lambda\left(1-e^{-(\beta / x)^{\alpha}}\right)}{\left[1+(\theta-1) e^{-(\beta / x)^{\alpha}}\right]^{3}}\right]^{n-1} .\right.
$$

\section{Characterizations}

In this section, the TG-IW distribution is characterized through: (i) Ratio of the truncated moments; (ii) the reverse hazard rate function and (iii) Elasticity function. 
Sri Lankan Journal of Applied Statistics, Vol (18-3)

We present our characterizations (i)- (iii) in three subsections.

\subsection{Characterization of the TG-IW Distribution through Ratio of Truncated Moments}

In this subsection, the TG-IW distribution is characterized using Theorem 1 (Glänzel; 1990) on the basis of two the truncated moments of X. Theorem 1 is given in Appendix A.

Proportion 6.1.1: Let $X: \Omega \rightarrow(0, \infty)$ be a continuous random variable. Let

$$
h_{1}(x)=\frac{(\alpha+m)}{\theta \alpha \beta^{\alpha} x^{m}} e^{(\beta / x)^{\alpha}}\left[1+(\theta-1) e^{-(\beta / x)^{\alpha}}+\lambda\left(1-e^{-(\beta / x)^{\alpha}}\right)\right]^{-1}\left[1+(\theta-1) e^{-(\beta / x)^{\alpha}}\right]^{3}
$$

and

$$
h_{2}(x)=\frac{(\alpha+m+1)}{\theta \alpha \beta^{\alpha} x^{(m+1)}} e^{(\beta / x)^{\alpha}}\left[1+(\theta-1) e^{-(\beta / x)^{\alpha}}+\lambda\left(1-e^{-(\beta / x)^{\alpha}}\right)\right]^{-1}\left[1+(\theta-1) e^{-(\beta / x)^{\alpha}}\right]^{3}, \mathrm{x}>0
$$

The pdf of $\mathrm{X}$ is (4) if and only if $p(x)$ in Theorem $\mathrm{G}$ has the form $p(x)=x$.

Proof. The pdf of $\mathrm{X}$ is (4). Now

$$
(1-F(x)) E\left[h_{1}(x) \mid X \geq x\right]=x^{-\alpha-m}
$$

And

$$
(1-F(x)) E\left[h_{2}(x) \mid X \geq x\right]=x^{-\alpha-m-1}, x>0 .
$$

After simplification, we have $\frac{E\left[\mathrm{~h}_{1}(X) \mid \mathrm{X} \geq \mathrm{x}\right]}{E\left[\mathrm{~h}_{2}(X) \mid \mathrm{X} \geq \mathrm{x}\right]}=p(x)=x$ and $p^{\prime}(x)=1$.

The differential equation $s^{\prime}(x)=\frac{p^{\prime}(x) h_{2}(x)}{p(x) h_{2}(x)-h_{1}(x)}=(\alpha+m+1) x^{-1}$ has solution $s(x)=(\alpha+m+1) \ln x$.

Now, in the light of theorem 1, X has pdf (4). 
Sri Lankan Journal of Applied Statistics, Vol (18-3)

Corollary 6.1.1: Let $X: \Omega \rightarrow(0, \infty)$ be a continuous random variable and

$$
h_{2}(x)=\frac{(\alpha+m+1)}{\theta \alpha \beta^{\alpha} x^{(m+1)}} e^{(\beta / x)^{\alpha}}\left[1+(\theta-1) e^{-(\beta / x)^{\alpha}}+\lambda\left(1-e^{-(\beta / x)^{\alpha}}\right)\right]^{-1}\left[1+(\theta-1) e^{-(\beta / x)^{\alpha}}\right]^{3}, \mathrm{x}>0 .
$$

The pdf of $\mathrm{X}$ is (4) if and only if functions $p(x)$ and $h_{1}(x)$ satisfy the equation

$$
\frac{p^{\prime}(x)}{p(x) h_{2}(x)-h_{1}(x)}=\theta \alpha \beta^{\alpha} x^{m} e^{-(\beta / x)^{\alpha}}\left[1+(\theta-1) e^{-(\beta / x)^{\alpha}}\right]^{-3}\left[1+(\theta-1) e^{-(\beta / x)^{\alpha}}+\lambda\left(1-e^{-(\beta / x)^{\alpha}}\right)\right] .
$$

Remarks 6.1.1: The answer of the above differential equation is

$$
p(x)=x^{(\alpha+m+1)} \int\left(-h_{1}(t) \theta \alpha \beta^{\alpha} x^{-(\alpha+1)} e^{-(\beta / x)^{\alpha}}\left[1+(\theta-1) e^{-(\beta / x)^{\alpha}}+\lambda\left(1-e^{-(\beta / x)^{\alpha}}\right)\right]\left[1+(\theta-1) e^{-(\beta / x)^{\alpha}}\right]^{-3}\right) d x+D
$$

where $\mathrm{D}$ is constant.

\subsection{Characterization via Reverse Hazard Function}

Here we characterize the TG-IW distribution via reverse hazard function of X.

Definition 6.2.1: Let $X: \Omega \rightarrow(0, \infty)$ be a continuous random variable having absolutely continuous cdf $F(x)$ and pdf $f(x)$ if and only if the reverse hazard function $r_{F}(x)$ is twice differentiable function fulfills the differential equation

$$
\frac{d}{d x}[\log f(x)]=\frac{r_{F}^{\prime}(x)}{r_{F}(x)}+r_{F}(x) .
$$

Proposition 6.2.1 Let $\mathrm{X}: \Omega \rightarrow(0, \infty)$ be continuous random variable .The pdf of $\mathrm{X}$ is (4) if and only if its reverse hazard function, $r_{F}$ fulfills the first order differential equation 
Sri Lankan Journal of Applied Statistics, Vol (18-3)

$r_{F}^{\prime}(x)\left[\left(1+(\theta-1) e^{-(\beta / x)^{\alpha}}\right)+\lambda\left(1-e^{-(\beta / x)^{\alpha}}\right)\right]-\alpha \beta^{\alpha} x^{-(\alpha+1)} e^{(-\beta / x)^{\alpha}}(\theta-1-\lambda) r_{F}(x)=-\alpha \beta x^{-\alpha-2}\left\lfloor-(1+\lambda)(\alpha+1)+\frac{2 \lambda \theta e^{-(\beta x / x)^{\alpha}}\left((\alpha+1)\left(1+(\theta-1) e^{-(\beta \gamma x)^{\alpha}}\right)-\alpha \beta^{\alpha} x^{-\alpha}\right)}{\left(1+(\theta-1) e^{-(\beta \beta x)^{\alpha}}\right)^{2}}\right\rfloor$

Proof: If the pdf of $X$ is (4), then the above differential equation holds. Now, if the differential equation holds, then

$$
\frac{d}{d x}\left\{r_{F}(x)\left[\left(1+(\theta-1) e^{-(\beta / x)^{\alpha}}\right)+\lambda\left(1-e^{-(\beta / x)^{\alpha}}\right)\right]\right\}=\alpha \beta^{\alpha} \frac{d}{d x} x^{-\alpha-1}\left(1+\lambda-\frac{2 \lambda \theta e^{-(\beta / x)^{\alpha}}}{\left(1+(\theta-1) e^{-(\beta / x)^{\alpha}}\right)}\right)
$$

or

$$
r_{F}(x)=\frac{\alpha \beta^{\alpha}\left(1+\lambda-\frac{2 \lambda \theta e^{-(\beta / x)^{\alpha}}}{\left(1+(\theta-1) e^{-(\beta / x)^{\alpha}}\right)}\right)}{x^{\alpha+1}\left[\left(1+(\theta-1) e^{-(\beta / x)^{\alpha}}\right)+\lambda\left(1-e^{-(\beta / x)^{\alpha}}\right)\right]}, x>0
$$

which is the reverse hazard function of the TG-IW distribution.

\subsection{Characterization via Elasticity Function}

Here we introduce characterization of the TG-IW distribution via elasticity.

Definition 6.3.1: Let $X: \Omega \rightarrow(0, \infty)$ be a continuous random variable having absolutely continuous $F(x)$ and pdf $f(x)$, if and only if the elasticity function $e_{F}(x)$, of a twice differentiable function fulfills the differential equation

$$
\frac{d}{d x}[\ln f(x)]=\frac{e_{F}^{\prime}(x)}{e_{F}(x)}+\frac{e_{F}(x)}{x}-\frac{1}{x}
$$

Proposition 6.3.1 Let $X: \Omega \rightarrow(0, \infty)$ be continuous random variable .The pdf of $X$ is (4) if and only if its elasticity, $e_{F}(x)$ fulfills the first order differential equation 
Sri Lankan Journal of Applied Statistics, Vol (18-3)

$$
\begin{aligned}
e_{F}^{\prime}(x)\left[\left(1+(\theta-1) e^{-(\beta / x)^{\alpha}}\right)+\lambda\left(1-e^{-(\beta / x)^{\alpha}}\right)\right\rfloor-\alpha \beta^{\alpha} x^{-(\alpha+1)} e^{-(\beta / x)^{\alpha}}(\theta-1-\lambda) e_{F}(x)= \\
\quad-\frac{\alpha^{2} \beta^{\alpha}}{x^{\alpha+1}\left(1+(\theta-1) e^{-(\beta / x)^{\alpha}}\right)^{2}}\left[2 \lambda \theta e^{-(\beta / x)^{\alpha}}\left(1+(\theta-1) e^{-(\beta / x)^{\alpha}}-x^{-\alpha} \beta^{\alpha}\right)-(1+\lambda)\left(1+(\theta-1) e^{-(\beta / x)^{\alpha}}\right)^{2}\right] .
\end{aligned}
$$

Proof: If the pdf of $X$ is (4), then the above differential equation holds. Now, if the differential equation holds, then

$$
\begin{aligned}
& \frac{d}{d x}\left\{e_{F}(x)\left[\left(1+(\theta-1) e^{-(\beta / x)^{\alpha}}\right)+\lambda\left(1-e^{-(\beta / x)^{\alpha}}\right)\right]\right\}=\alpha \beta^{\alpha} \frac{d}{d x} x^{-\alpha}\left(1+\lambda-\frac{2 \lambda \theta e^{-(\beta / x)^{\alpha}}}{\left(1+(\theta-1) e^{-(\beta / x)^{\alpha}}\right)}\right), \\
& \text { or } \quad \alpha \beta^{\alpha}\left(1+\lambda-\frac{2 \lambda \theta e^{-(\beta / x)^{\alpha}}}{\left(1+(\theta-1) e^{-(\beta / x)^{\alpha}}\right)}\right) \\
& e_{F}(x)=\frac{x^{\alpha}\left[\left(1+(\theta-1) e^{-(\beta / x)^{\alpha}}\right)+\lambda\left(1-e^{-(\beta / x)^{\alpha}}\right)\right]}{x>0,}
\end{aligned}
$$

which is the elasticity function of the TG-IW distribution.

\section{Maximum Likelihood Estimation}

The parameter estimates are derived with maximum likelihood method for TG-IW distribution. The log-likelihood function for the TG-IW the parameters vector $\Phi=(\alpha, \beta, \theta, \lambda)$ is

$$
\begin{aligned}
L\left(x_{i}, \Phi\right)=n \log \theta+n \log \alpha+n \alpha \log \beta & -(\alpha+1) \sum \log x_{i}-\beta^{\alpha} \sum x_{i}^{-\alpha} \\
& -2 \sum \log \left[1+(\theta-1) e^{-\left(\beta / x_{i}\right)^{\alpha}}\right]+\sum \log \left[1+\lambda-\frac{2 \lambda \theta e^{-\left(\beta / x_{i}\right)^{\alpha}}}{\left(1+(\theta-1) e^{-\left(\beta / x_{i}\right)^{\alpha}}\right)}\right] .
\end{aligned}
$$

In order to compute the estimates of parameters of TG-IW distribution, the following nonlinear equations must be solved simultaneously: 
Sri Lankan Journal of Applied Statistics, Vol (18-3)

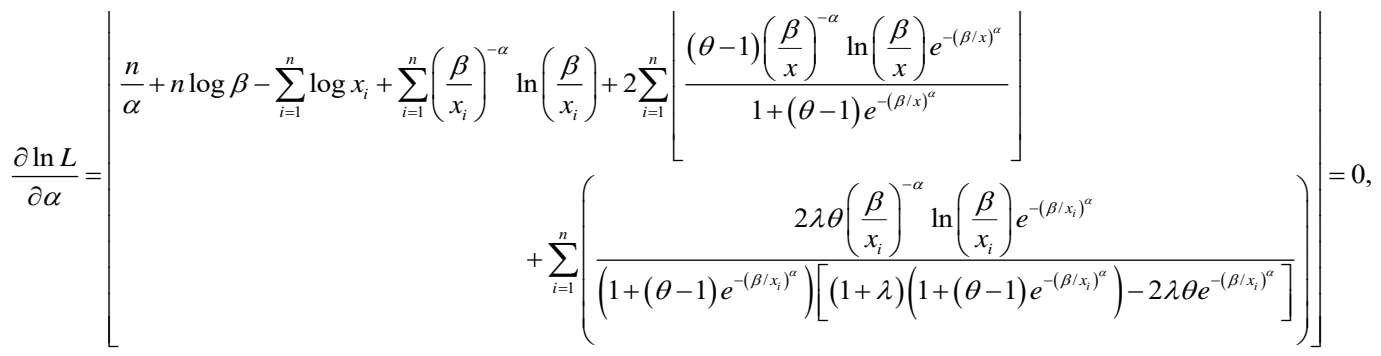

$$
\begin{aligned}
& \frac{\partial \ln L}{\partial \beta}=\left\lfloor\begin{array}{rl}
\frac{n \alpha}{\beta}-\alpha \beta^{\alpha-1} \sum_{i=1}^{n} x_{i}^{-\alpha}+2(\theta-1) & \sum_{i=1}^{n}\left\lfloor\frac{e^{-\left(\beta / x_{i}\right)^{\alpha}} \alpha \beta^{\alpha-1} x_{i}^{-\alpha}}{1+(\theta-1) e^{-\left(\beta / x_{i}\right)^{\alpha}}}\right\rfloor \\
& \left.\left.+\sum_{i=1}^{n} \frac{2 \lambda \theta e^{-\left(\beta / x_{i}\right)^{\alpha}} \alpha \beta^{\alpha-1} x_{i}^{-\alpha}}{\left(1+(\theta-1) e^{-\left(\beta / x_{i}\right)^{\alpha}}\right)\left[(1+\lambda)\left(1+(\theta-1) e^{-\left(\beta / x_{i}\right)^{\alpha}}\right)-2 \lambda \theta e^{-\left(\beta / x_{i}\right)^{\alpha}}\right.}\right]\right\rfloor=0,
\end{array}\right\rfloor \\
& \frac{\partial \ln L}{\partial \theta}=\frac{n}{\theta}-2 \sum_{i=1}^{n}\left[\frac{e^{-\left(\beta / x_{i}\right)^{\alpha}}}{1+(\theta-1) e^{-\left(\beta / x_{i}\right)^{\alpha}}}\right]-\sum_{i=1}^{n} \frac{\left(1+(\theta-1) e^{-\left(\beta / x_{i}\right)^{\alpha}}\right) 2 \lambda e^{-(\beta / x)^{\alpha}}-2 \lambda \theta e^{-2\left(\beta / x_{i}\right)^{\alpha}}}{\left(1+(\theta-1) e^{-\left(\beta / x_{i}\right)^{\alpha}}\right)^{2}\left[1+\lambda-\frac{2 \lambda \theta e^{-\left(\beta / x_{i}\right)^{\alpha}}}{\left(1+(\theta-1) e^{-\left(\beta / x_{i}\right)^{\alpha}}\right)}\right]}=0, \text { (35) } \\
& \frac{\partial \ln L}{\partial \lambda}=\sum_{i=1}^{n}\left[1-\frac{2 \theta e^{-\left(\beta / x_{i}\right)^{\alpha}}}{\left(1+(\theta-1) e^{-\left(\beta / x_{i}\right)^{\alpha}}\right)}\right]\left[1+\lambda-\frac{2 \lambda \theta e^{-\left(\beta / x_{i}\right)^{\alpha}}}{\left(1+(\theta-1) e^{-\left(\beta / x_{i}\right)^{\alpha}}\right)}\right]^{-1}=0 .
\end{aligned}
$$

\subsection{Application: Times of Failure and Running Times for 30 Units from Eld- Tracking Study}

The TG-IW distribution is compared with T-IW, G-IW and IW distributions. Different goodness fit measures like Cramer-von Mises (W), Anderson Darling (A), Kolmogorov- Smirnov statistics, Akaike information criterion (AIC), consistent Akaike information criterion (CAIC), Bayesian information criterion (BIC), Hannan-Quinn information criterion (HQIC) and likelihood ratio statistics are computed using R-package for times of failure and running times (Meeker and Escobar ; 1998) for 30 units from eld-tracking. The values of data are: $2.75,0.13$, $1.47,0.23,1.81,0.30,0.65,0.10,3.00,1.73,1.06,3.00,3.00,2.12,3.00,3.00,3.00$, $0.02,2.61,2.93,0.88,2.47,0.28,1.43,3.00,0.23,3.00,0.80,2.45$, and 2.66.

The better fit corresponds to smaller W, A, K-S, AIC, CAIC, BIC, HQIC and- $\ell$ 
value. The maximum likelihood estimates (MLEs) of unknown parameters and values of goodness of fit measures are computed for the TG-IW distribution and its sub-models. The MLEs and goodness-of-fit statistics like $\mathrm{W}$ and $\mathrm{A}$ are given in table 2 . Table 3 displays goodness-of-fit values.

Table 2: MLEs and Goodness-of-Fit Statistics for Times of Failure and Running Times

\begin{tabular}{|l|l|l|l|l|l|l|}
\hline Model & $\alpha$ & $\beta$ & $\theta$ & $\lambda$ & $\mathrm{W}$ & $\mathrm{A}$ \\
\hline $\begin{array}{l}\text { TG- } \\
\text { IW }\end{array}$ & 1.316772419 & 0.034439749 & 0.003439308 & 0.979107526 & 0.3304939 & 2.119582 \\
\hline T-IW & 0.6251490982 & 0.5399117441 & & 0.0000000001 & 0.5541168 & 3.02536 \\
\hline G-IW & 1.385703702 & 0.02644853 & 0.004433838 & ------------- & 0.4119026 & 2.327227 \\
\hline IW & 0.6251527 & 0.5399035 & & & 0.5541174 & 3.025363 \\
\hline
\end{tabular}

Table 3: Goodness-of-Fit Statistics for Times of Failure and Running Times

\begin{tabular}{|l|l|l|l|l|l|l|}
\hline Model & D $(\mathrm{KS})$ & AIC & CAIC & BIC & HQIC & $-\ell$ \\
\hline TG-IW & 0.206 & 107.5886 & 109.1886 & 113.1934 & 109.3816 & 49.7943 \\
\hline T-IW & 0.2899 & 126.5836 & 127.5067 & 130.7872 & 127.9284 & 60.2918 \\
\hline G-IW & 0.2429 & 110.1996 & 111.1226 & 114.4031 & 111.5443 & 52.09978 \\
\hline IW & 0.2899 & 124.5836 & 125.028 & 127.386 & 125.4801 & 60.2918 \\
\hline
\end{tabular}

The TG-IW distribution is best fitted than T-IW, G-IW and IW distribution because the values of all criteria are smaller for the TG-IW distribution.

\section{Conclusions}

We have developed a more flexible the TG-IW distribution that is suitable for applications in survival analysis, reliability and actuarial science. The important properties of the TG-IW distribution like survival function, hazard function, reverse hazard function, cumulative hazard function, Mills ratio, elasticity, quantile function, moments about origin, negative moments, fractional moments, moment generating function, Cumulants, incomplete moments, inequality measures, residual and reversed residual life functions, order statistics and many other properties are presented.

The TG-IW distribution is characterized through ratio of truncated moments, reverse hazard rate function and elasticity function. Maximum likelihood estimates 
are computed. Goodness of fit shows that the TG-IW distribution is a better fit. An application of the TG-IW model to times of failure and running times for 30 units from eld-tracking is illustrated to show significance and flexibility of the TG-IW distribution.

\section{References}

1. Afify, A. Z., Alizadeh, M.,Yousof, H. M., Aryal, G., and Ahmad, M., (2016). The Transmuted Geometric-G Family of Distributions: Theory and Applications. Pak. J. Statist, 32(2), 139-160.

2. Afify, A. Z., Cordeiro, G. M., Yousof, H. M., Alzaatreh, A., \& Nofal, Z. M., (2016). The Kumaraswamy transmuted-G family of distributions: properties and applications. Journal of Data Science, 14(2), 245-270.

3. Alexander, C., Cordeiro, G.M., Ortega, E.M.M. and Sarabia, J.M., (2012). Generalized beta generated distributions. Computational Statistics and Data Analysis, 56, 1880-1897.

4. Alizadeh, M., Tahir, M. H., Cordeiro, G. M., Mansoor, M., Zubair, M., \&Hamedani, G. G. (2015). The Kumaraswamy Marshal-Olkin family of distributions. Journal of the Egyptian Mathematical Society, 23(3), 546557.

5. Alizadeh, M., Emadi, M., Doostparast, M., Cordeiro, G.M., Ortega, E.M.M. and Pescim, R.R., (2015).Kumaraswamy odd log-logistic family of distributions: Properties and applications. Hacettepe Journal of Mathematics and Statistics.

6. Azzalini A., (1985).A class of distributions which includes the normal ones. Scand J Statist., 12, 171-178.

7. Bhatti, F.A., (2017). Some Characterizations of Inverse Weibull-Geometric Distribution, Int. J. Modern Math. Sci., 15 (4), pp.424-432.

8. Bhatti, F. A., Hamedani, G. G., Korkmaz, M. Ç., \& Ahmad, M. (2018). The transmuted geometric-quadratic hazard rate distribution: development, properties, characterizations and applications. Journal of Statistical Distributions and Applications, 5(1), 4.

9. Cordeiro, G. M., Alizadeh, M., Tahir, M. H., Mansoor, M., Bourguignon, M., \&Hamedani, G. G., ( 2016). The beta odd log-logistic generalized family of distributions. Hacet. J. Math. Stat, 45, 1175-1202.

10. Cordeiro, G.M. and de Castro, M., (2011). A new family of generalized distributions. Journal of Statistical Computation and Simulation, 81, 883898. 
11. Eugene N., Lee C. and Famoye F. (2002). Beta-normal distribution and its applications. Commun Statist Theor Meth. 31,497-512.

12. Glänzel W.A., (1986). Characterization theorem based on truncated moments and its application to some distribution families, Mathematical Statistics and Probability Theory (Bad Tatzmannsdorf, Vol. B, Reidel, Dordrecht, 1987; 75-84.

13. Gupta R.C., Gupta P.I. and Gupta R.D.. Modeling failure time data by Lehmann alternatives. Commun Statist Theor Meth., 27, 887-904(1998).

14. Jones M.C., (2004). Families of distributions arising from the distributions of order statistics. Test, 13, 1-43.

15. Kandil, A.E.F. Mohamed, M. Kayid, and M. M. R. Mahdy. Median inactivity time function and its reliability properties." Economic Quality Control, 25(2), 253-268(2010).

16. KhanAdil H. and Jan T.R., (2016). Inverse Weibull-Geometric Distribution, Int. J. Modern Math. Sci., 14 (2),pp.134-146.

17. Marshall, A.W. and Olkin, I., (1997). A new method for adding a parameter to a family of distributions with application to the exponential and Weibull families. Biometrika, 84, 641-652.

18. Meeker W. Q., and Escobar L. A., (1998). Statistical Methods for Reliability Data. John Wiley, New York, 383.

19. Nofal, Z. M., El Gebaly, Y. M., Altun, E., Alizadeh, M., \& Butt, N. S., (2017). The Transmuted Geometric-Weibull distribution: Properties, Characterizations and Regression Models. Pakistan Journal of Statistics and Operation Research, 13(2), 395-416.

20. Shaw, W.T. and Buckley, I.R.C., (2007).The alchemy of probability distributions: beyond Gram-Charlier expansions and a skew-kurtoticnormal distribution from a rank transmutation map. Research Report.

21. Torabi H. and Montazari N.H., (2014). The logistic-uniform distribution and its application. Commun Statist Simul. Comput., 43, 2551-2569.

22. Zografos K. and Balakrishnan, N.,(2009). On families of beta and generalized gamma generated distributions and associated inference. Statistical Methodology, 6, 344-362.

\section{Appendix A}


Sri Lankan Journal of Applied Statistics, Vol (18-3)

Theorem 1: Let $(\Omega, F, P)$ be a probability space and let $\left[d_{1}, d_{2}\right]$ be an interval with $d_{1}<d_{2}\left(d_{1}=-\infty, d_{2}=\infty\right)$. Supposing that a continuous random variable $X: \Omega \rightarrow\left[\mathrm{d}_{1}, \mathrm{~d}_{2}\right]$ have distribution function $\mathrm{F}$. Real functions $h_{1}(x)$ and $h_{2}(x)$ are continuous on $\left[\mathrm{d}_{1}, \mathrm{~d}_{2}\right]$ such that $\frac{E\left[\mathrm{~h}_{1}(X) / \mathrm{X} \geq \mathrm{x}\right]}{E\left[\mathrm{~h}_{2}(X) / \mathrm{X} \geq \mathrm{x}\right]}=p(x)$ is real function $p(x)$ and should be in simple form. Assume that $h_{1}, h_{2} \varepsilon C\left(\left[\mathrm{~d}_{1}, \mathrm{~d}_{2}\right]\right)$, $p(x) \varepsilon C^{2}\left(\left[\mathrm{~d}_{1}, \mathrm{~d}_{2}\right]\right)$ and $\mathrm{F}$ is twofold continuously differentiable and strictly monotone function on the $\operatorname{set}\left[\mathrm{d}_{1}, \mathrm{~d}_{2}\right]$ : To end, adopt that the relation $h_{2}(x) p(x)=h_{1}(x)$ has no real solution in the interior of $\left[\mathrm{d}_{1}, \mathrm{~d}_{2}\right]$.

Then $\mathrm{F}$ is attained from the functions $h_{1}(x), h_{2}(x)$ and $s(x)$ as $F(x)=\int_{\ln k}^{x} K\left|\frac{p^{\prime}(t)}{p(t) h_{2}(t)-h_{1}(t)}\right| \exp (-s(t)) d t$, where $s(t)$ is obtained from equation $s^{\prime}(t)=\frac{p^{\prime}(t) h_{2}(t)}{p(t) h_{2}(t)-h_{1}(t)}$ and $\mathrm{K}$ is a fixed number, preferred to make $\int_{d_{1}}^{d_{2}} d F=1$ 